\title{
Energy confinement in steady-state ELMy H-modes in JET
}

\author{
J G Cordey ${ }^{1}$, D C McDonald ${ }^{1}$, K Borrass ${ }^{2}$, M Charlet ${ }^{1}$, I Coffey ${ }^{1}$, \\ A Kallenbach ${ }^{2}$, K Lawson ${ }^{1}$, P Lomas ${ }^{1}$, J Ongena ${ }^{3}$, J Rapp ${ }^{4}$, F Ryter ${ }^{2}$,

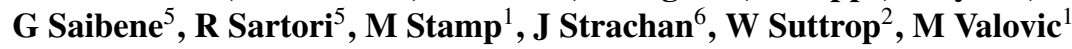 \\ and contributors to the EFDA-JET Workprogramme ${ }^{7}$
}

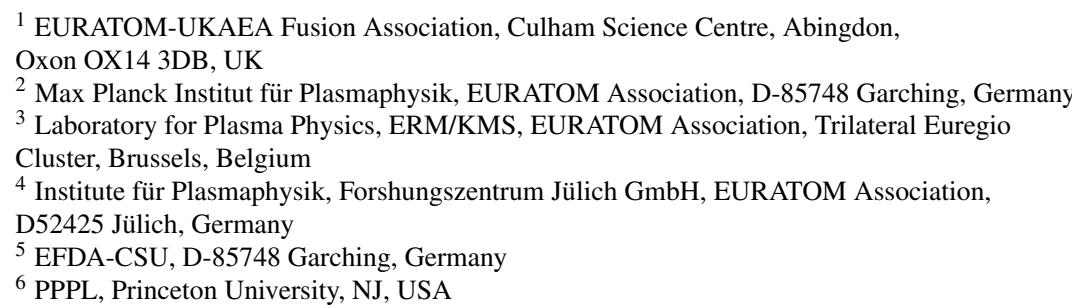

Received 10 April 2002, in final form 16 May 2002

Published 27 August 2002

Online at stacks.iop.org/PPCF/44/1929

\begin{abstract}
It is shown statistically that, divertor closure, plasma shaping and density peaking improve the energy confinement of ELMy H-modes, whilst the confinement degrades as the Greenwald density limit is approached. A prediction of the influence of these effects on the next step device ITER is given.
\end{abstract}

\section{Introduction}

During the last seven years an extensive confinement database has been assembled on JET of steady-state ELMy H-mode plasmas. The database was started under the JET Joint Undertaking and has been continued under EFDA with the addition of a further 200 pulses. In this paper the database is used to assess the effect of three parameters upon the energy confinement: plasma shaping, the proximity of the density to the Greenwald density limit and the peaking of the density profile. There is clear evidence from single parameter scans that these three variables do influence the confinement; however, the present scaling expression, used to predict the performance of ITER, namely IPB98 $(y, 2)[1]$, does not contain these variables.

In the remainder of this paper, in section 2.1 we first analyse the full ELMy H-mode database and determine the scaling of the confinement with the plasma shaping and the vicinity

7 See annex of Pamela J et al 2001 Overview of recent JET results and future perspectives Proc. 18th Int. Conf. on Fusion Energy 2000 (Sorrento, 2000) (Vienna: IAEA). 
to the Greenwald limit, then in section 2.2 a reduced database, which contains pulses with reliable density peaking measurements, is analysed, to assess the effect on energy confinement of density peaking. Finally, in section 3 prediction of these effects in the next step device ITER is given.

\section{Analysis of steady-state database}

\subsection{Full database}

The steady-state ELMy H-mode database contains some 1248 pulses and includes a wide range of current $(1 \mathrm{MA}<I<4.5 \mathrm{MA})$, toroidal field $(1 \mathrm{~T}<B<3.8 \mathrm{~T})$ and isotopes $\mathrm{H}$, D, D-T, T. Recently a substantial quantity of high-density data has been obtained close to the Greenwald limit $\left(n_{\mathrm{GR}}=I / \pi a^{2}\right)$, such that the present database now contains pulses with $0.2<n / n_{\mathrm{GR}}<1.2$, where $n$ is the central line-average density. The higher densities being obtained by employing sophisticated gas fuelling and power control techniques [2-6]. There are no pulses in which pellets are injected or pulses with internal transport barriers (ITBs). There are both Types I and III ELMs and a wide range of configurations with upper triangularity $\delta_{\mathrm{u}}$ ranging from $0<\delta_{\mathrm{u}}<0.7$, and the lower triangularity $0.2<\delta_{\mathrm{L}}<0.5$ and three divertor types Marks I, II and the gas box MarkGB. The most closed divertor amongst three is the gas box, both the Marks I and II being open divertors.

The data are compared with the IPB98 $(\mathrm{y}, 2)$ scaling, which has the form

$$
\tau_{\varepsilon 98}=0.0562 I^{0.93} B^{0.15} n^{0.41} P^{-0.69} M^{0.19} R^{1.97} \varepsilon^{0.58} \kappa_{\mathrm{a}}^{0.78}
$$

in figure $1(a)$. Here $H_{98}\left(\equiv \tau_{\varepsilon} / \tau_{\varepsilon 98}\right)$ is shown versus the density divided by the Greenwald density.

The data have been grouped by plasma current and one can see that in the present dataset only the lower current data $I<2.5 \mathrm{MA}$ achieves a density above the Greenwald limit. The reason for the absence of the high-current data with $n>n_{\mathrm{GR}}$ is thought to be due to the lack of available input power, rather than a fundamental limit [7]. The argument being that to achieve high densities it is essential to have Type I ELMs, and to maintain these ELMs the input power has to exceed the threshold power by at least a factor of 2 . The $H_{98}$ factor for the full JET dataset, Types I and III ELMs, is 0.92 , for Types I ELMs only is 0.95 and for Type III ELMs only is 0.87 . There are marginally more Type I ELM pulses (672) than Type III ELMy pulses (576) in the database.

We first examine whether there is any dependence of the energy confinement on the divertor type, by calculating the dependence of the residuals with respect to the IPB98(y, 2) scaling upon the divertor type. For this particular assessment, we restrict the datasets to the region where there is a substantial overlap in the data from the three divertors, this is the region $0.2<\delta<0.4,0.2<n / n_{\mathrm{GR}}<1.0$ and Type I ELMs only. The Marks I and II divertors are found to have the same average residual ( $H$ factor) with respect to the IPB98(y, 2) scaling; however, the gas box is found to have an $8 \%$ higher $H_{98}$ factor, as shown in figure $1(b)$. This difference is also seen in all data subsets, such as $0.4<n / n_{\mathrm{GR}}<0.8$, and hence is not due to the choice of dataset. The reason for the apparent improved confinement in the gas box though is not understood. Previous experiments in ASDEX with a closed divertor also gave rise to improved confinement, so it is not the first time this effect has been observed. Experiments will be completed in 2002 with the Septum removed, which gives a more open divertor, and it will be interesting to see whether the confinement of these plasmas is then degraded over that of the gas box. Anyway in all future analysis this difference in the divertor type is allowed for by introducing a factor $(1+0.08$ div $)$ into the IPB98 $(y, 2)$ scaling expression where div $=0$ for Marks I and II and div $=1$ for the gas box data. 

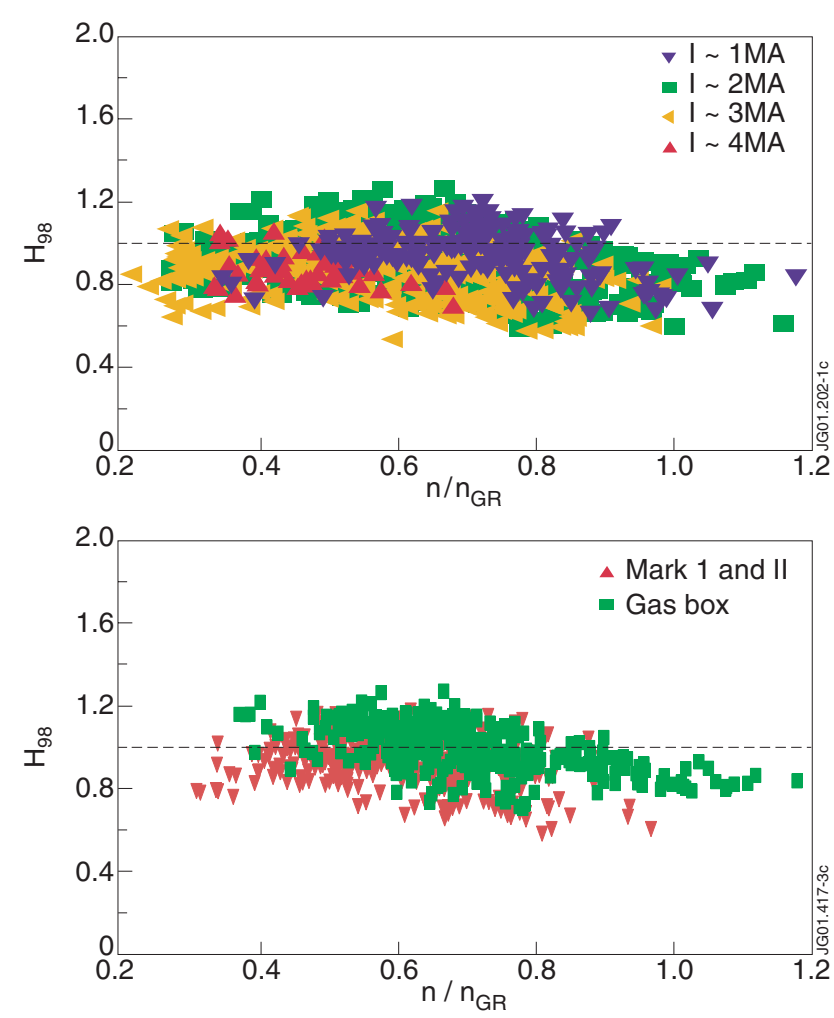

Figure 1. $H_{98}\left(\equiv \tau_{\varepsilon} / \tau_{\varepsilon} 98\right)$ versus $n / n_{\mathrm{GR}}$. The data are grouped by $(a)$ current $I$ in MA $0.5<I<1.5,1.5<I<2.5,2.5<I<3.5$ and $I>3.5$ and $(b)$ divertor type. Note that the Type I dataset is shown.

Turning to the dependence of the residuals or $H$ factor on shaping and the vicinity to the Greenwald limit, we find that the $H$ factor increases with triangularity and degrades as the Greenwald limit is approached. The form is

$$
H\left(\equiv \frac{\tau}{\tau_{\varepsilon 98}^{\prime}}\right)=0.95 \pm 0.016+(0.37 \pm 0.062) \delta-(0.25 \pm 0.022) \frac{n}{n_{\mathrm{GR}}},
$$

where $\tau^{\prime}{ }_{\varepsilon 98}$ is equation (1) multiplied by the divertor factor $(1+0.08 \mathrm{div})$.

Although the RMSE of this fit (13.32\%) is smaller than that (13.95\%) in the absence of the triangularity and Greenwald terms, the reduction is not very large.

There are several other possible combinations of $\delta$ and $\kappa$ that could be used to describe the plasma shape and one that gives a significantly reduced RMSE is the parameter $q_{\mathrm{f}}\left(\equiv q_{95} / q_{\text {cyl }}\right.$ where $\left.q_{\text {cyl }}=5 a^{2} \kappa_{\mathrm{a}} B / R I\right)$ used by Kardaun et al [8] in the study of pedestal behaviour. Replacing $\delta$ with $\ln q_{\mathrm{f}}$ and refitting the residuals gives

$$
H=(0.56 \pm 0.027)+(1.47 \pm 0.08) \ln q_{\mathrm{f}}-(0.25 \pm 0.02) \frac{n}{n_{\mathrm{GR}}},
$$

with an RMSE $=11.97 \%$. One should also note that the coefficient of $\ln q_{\mathrm{f}}$ is 18 standard errors whilst the coefficient of $\delta$ is only six standard errors. Hence it appears that $q_{\mathrm{f}}$ is a more useful parameter in describing the dependence of confinement on shape than triangularity, and we shall use this parameter in the remainder of the paper. This may be a consequence of the 
fact that $q_{\mathrm{f}}$ is closely related to the shear in the edge region which is stabilizing for the MHD modes which generate the ELMs.

\subsection{Reduced database}

To investigate the role of density peaking on confinement we have to extract a subset from the above dataset for which accurate values of the pedestal density are available. The pedestal density is obtained from the outer interferometer vertical line integral. Only pulses in which the top of the pedestal is intersected by the interferometer line of sight are retained, and furthermore only those in which the line average has been flagged as being of good quality are selected. We also restrict the dataset to Type I ELMs only, to avoid those pulses close to the L-H transition. The above selection reduces the dataset to 436 pulses from the original 1248 pulses. The main reduction is coming from the requirement to obtain an accurate line-average density in the edge region. An example of a pulse in which the above criteria are satisfied is shown in figures 2 and 3. This particular pulse is a 1.6 MA, 1.7 T ELMy H-mode pulse with a low gas input. Figures 2 and 3 show that as time evolves, the density profile slowly peaks whilst the edge density stays fairly constant. The line-average density exceeds the Greenwald limit in the latter part of the pulse, with the $H_{98}$ factor remaining approximately constant.

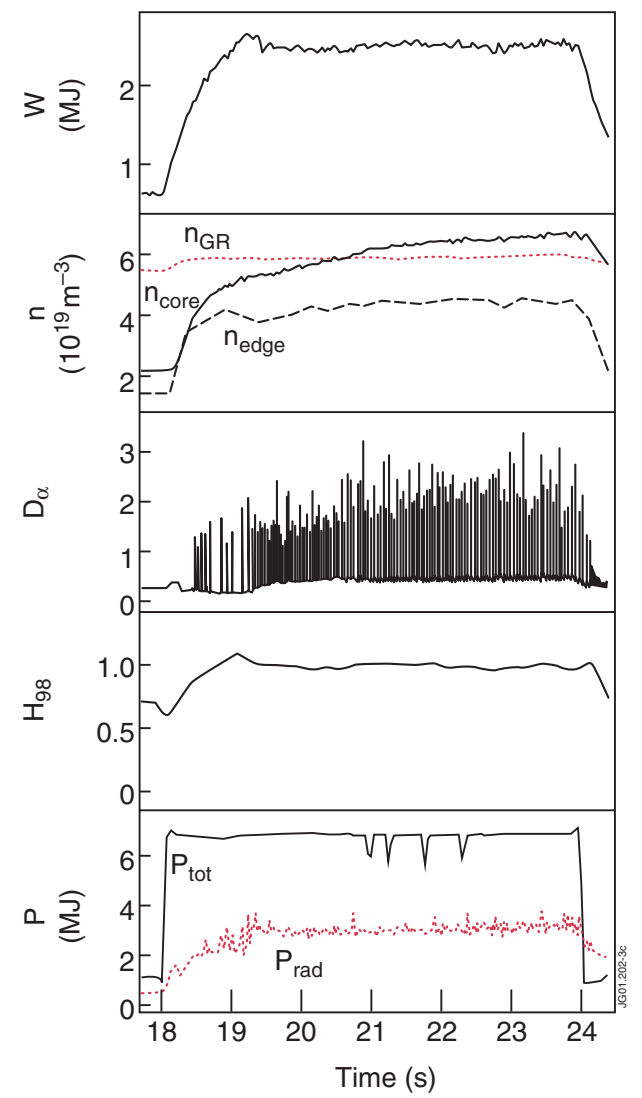

Figure 2. Time evolution of a typical pulse 48276. The traces are: (1) the stored energy; (2) the core line-average density, the edge line-average density $(R=3.78)$ and the Greenwald density; (3) the $D_{\alpha}$ emission; (4) the $H_{98}$ ratio and (5) the total power input and the radiated power. 


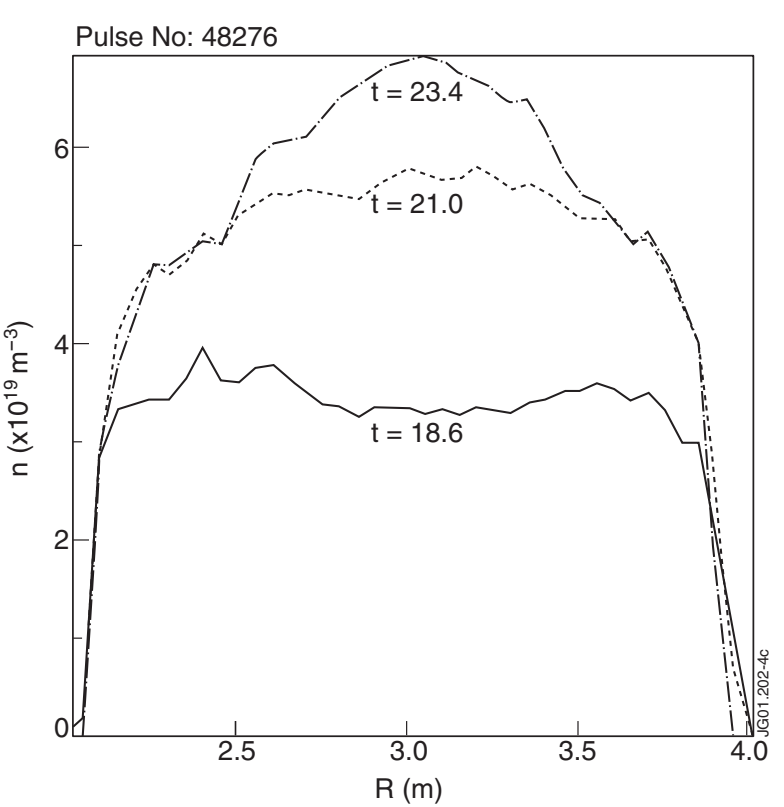

Figure 3. Electron density profile at three times.

We first examine the database for correlations between density and shaping. Figure 4 shows the density normalized to Greenwald density versus the shaping factor $q_{\mathrm{f}}$, with the data again grouped by current. From this figure it can be seen that there is only a weak correlation between the density and shaping in the $3 \mathrm{MA}$ dataset; for the 1 and $2 \mathrm{MA}$ dataset there is no correlation. This is due to the fact that fairly high densities can be obtained even in low-triangularity plasmas by carefully tuning the gas fuelling or by the injection of impurities to increase the edge radiation and reduce the deleterious effect of the ELMs. These type of experiments have yet to be repeated at higher currents.

Fitting the residuals of the $H$ factor as in section 2.1 with respect to the shaping factor $q_{\mathrm{f}}$, the Greenwald fraction $n / n_{\mathrm{GR}}$ and the density profile peaking $n / n_{\text {ped }}$, where $n$ is the line-average density through the plasma centre and $n_{\text {ped }}$ is the line-average density in the edge region, gives

$$
H_{\text {fit }}=0.46+1.35 \ln q_{\mathrm{f}}-0.17 \frac{n}{n_{\mathrm{GR}}}+0.38\left(\frac{n}{n_{\mathrm{ped}}}-1\right),
$$

with an RMSE of 9.6\%. The fit is shown in figure 5. This should be compared with an RMSE $=10.4 \%$ in the absense of the peaking term. Thus from equation (4) we see that density peaking improves the confinement; the effect, however, is not very large, compared to the shaping term. For example, for a typical value of $n / n_{\text {ped }} \sim 1.3$ in JET we see that the peaking would contribute 0.11 to the $H$ factor, whilst for a typical value of 0.4 for $\ln q_{\mathrm{f}}$ the contribution to $H$ would be 0.55 . Hence, the shaping is clearly the most dominant term.

Another interesting observation comparing figures $1(a)$ and 5 is that the degradation of the $4 \mathrm{MA}$ data with $n / n_{\mathrm{GR}}$ seen in figure 1( $a$ ) has been removed in figure 5 , indicating that it is the low value of $q_{\mathrm{f}}$, i.e. weak shaping and the flat density profile of the high current data that is responsible for the degradation with respect to the IPB98(y, 2) scaling. It is the intention to go to higher currents in strongly shaped plasmas in the experimental campaigns in 2003. 


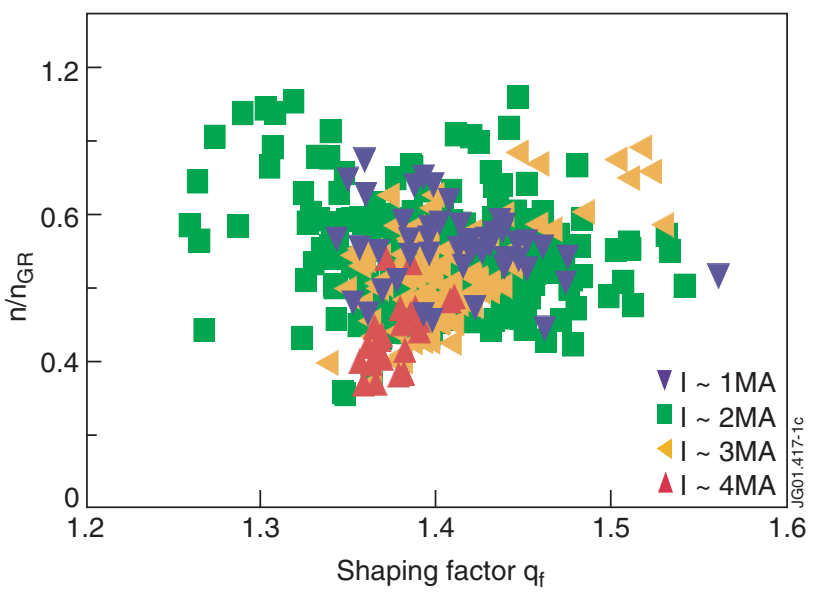

Figure 4. $n / n_{\mathrm{GR}}$ versus the shaping factor $q_{\mathrm{f}}$, current grouping as in figure 1 .

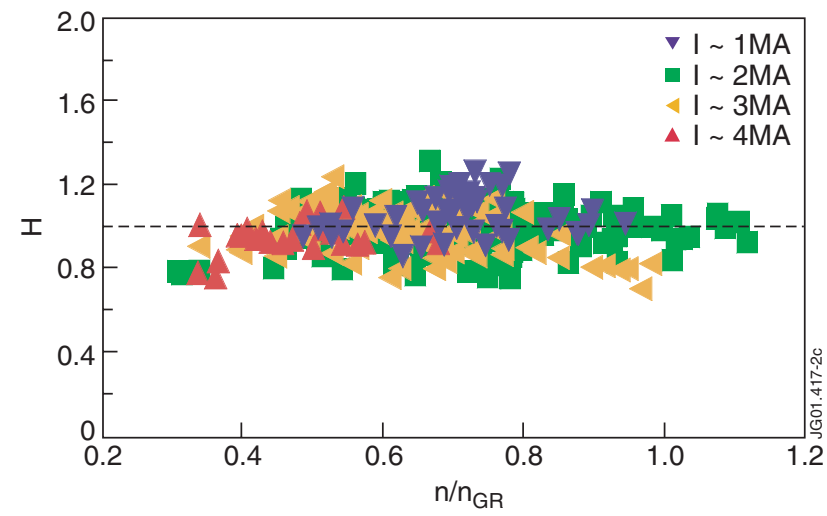

Figure 5. $H\left(\equiv \tau_{\varepsilon} / H_{\text {fit }} \tau_{98}^{\prime}\right)$ versus $n / n_{\mathrm{GR}}$, where $H_{\text {fit }}$ is given by equation (4).

\section{ITER predictions and conclusions}

Using equations (3) and (4), one can make a prediction for the $H_{98}$ factor in ITER. If we assume that in ITER $q_{\mathrm{f}}=1.58$, the operational density is $n / n_{\mathrm{GR}}=0.85$, and there is no peaking, then the $H_{98}$ factors from equations (3) and (4) are, respectively, $H_{98}=1.02$ and 0.94 . With a modest peaking factor $n / n_{\text {ped }}=1.3$, equation (4) gives an improved $H_{98}$ factor of 1.05 . For a closed divertor these factors may be increased by a further $8 \%$.

In summary we have shown that the plasma shaping is best described by the factor $q_{\mathrm{f}}$ ( $\equiv q_{95} / q_{\text {cyl }}$ ) rather than the triangularity. Increasing the shaping factor $q_{\mathrm{f}}$ improves the energy confinement. The confinement degrades as the Greenwald density limit is approached, but improves with density peaking. The most dominant effect is the shaping and this should certainly be included in future fits to the multi-machine database and in preditions of the performance of next step devices. 


\section{Acknowledgments}

This work was carried out under the European Fusion Development Agreement and was partly funded by Euratom and the UK Department of Trade and Industry.

\section{References}

[1] ITER Physics Basis 1999 Nucl. Fusion 392204

[2] Lomas P et al 2000 Plasma Phys. Control. Fusion 42 B115

[3] Maggi C 2001 Proc. 28th European Physical Society (EPS) Conf. on Controlled Fusion and Plasma Physics (Madeira, Portugal, June 2001)

[4] Saibene G et al Nucl. Fusion 39

[5] Saibene G et al 2001 Proc. 28th European Physical Society(EPS) Conf. on Controlled Fusion and Plasma Physics (Madeira, Portugal, 2001)

[6] Valovic Met al 2001 Proc. 28th European Physical Society(EPS) Conf. on Controlled Fusion and Plasma Physics (Madeira, Portugal, 2001)

[7] Sartori R et al 2001 Proc. 28th European Physical Society (EPS) Conf. on Controlled Fusion and Plasma Physics (Madeira, Portugal, 2001)

[8] Kardaun O et al 2000 IAEA Fusion Energy Conf. (Sorrento, Italy, 2000) 